\title{
Experimental Comparison of Orthogonal Moments as Feature Extraction Methods for Character Recognition
}

\author{
Miguel A. Duval, Sandro Vega-Pons, and Eduardo Garea \\ Advanced Technology Application Center (CENATAV), Havana, Cuba \\ \{mduval, svega, egarea\} @cenatav.co.cu
}

\begin{abstract}
The selection of a good feature extraction technique is very important in any classification problem. Moments, especially orthogonal moments, seem to be a powerful option in the case of digital image compression, description and recognition. Nowadays, there is a considerable amount of orthogonal moments reported in the literature, each one with some advantages and drawbacks. In this paper, we carry out an experimental comparison of several orthogonal moments for the character recognition problem. Firstly, we compare orthogonal moments with other kinds of feature extraction methods and after that, we compare the different orthogonal moments taking into account different evaluation parameters. Experiments were made by using printed and handwritten digit datasets and the well-known measures: precision, recall and accuracy were used to validate the results. This experimental study corroborates the good performance of orthogonal moments. Besides, more specific results obtained in different kinds of experimentations allow coming to conclusions that could be very useful for the community of image recognition practitioners.
\end{abstract}

Keywords: Orthogonal moments, Feature extraction, Character recognition.

\section{Introduction}

Classifier effectiveness depends, in a great way, on the feature collection used to describe the involved objects. This way, the selection of a suitable feature extraction method is very important for any particular classification problem. In the case of digital images, feature extraction methods are responsible for describing the images by extracting their main attributes for a classification process. It is desirable that features extracted from images be:

- Robust: They should be low sensitive to noise, bad illumination and other adverse factors that may be present in the original image.

- Discriminative: They should have the ability to distinguish images of different classes.

- Invariant: They should be invariant to some properties like translation, rotation and scale.

A large number of feature extraction methods are reported in the literature. They can be divided in two categories. The first one, called geometric features, includes 
moments, histograms, and direction features, while the second one, called structural features, includes Fourier descriptors, line element and topological features [1]. Some of the most popular feature extraction methods reported in the literature are the standard and elliptic Fourier descriptors [1]. They, together with curvature approximation [2], are used to describe figure shapes.

In this paper, we center our attention on the study of moments, particularly the orthogonal moments. Moments have been used as pattern features in numerous applications for 2D image recognition. It is well known their ability to extract global characteristics from the images like: shape area, center of mass, moment of inertia, and so on. Recently, new orthogonal moments such as Krawtchouk [3], dual Hahn [4] and Racah [5] have been introduced. Nowadays, there is a considerable amount of orthogonal moments reported in the literature. Each one of them presents some intrinsic properties that could be desirable or not for a particular problem. In this paper, we make an experimental comparison of the existing orthogonal moments for the character recognition problem. Firstly, we compare orthogonal moments with other kinds of feature extraction methods. After that, we carry out a comparative study of the stateof-the-art orthogonal moments taking into account different comparison parameters.

The rest of this paper is organized as follow: A group of most used orthogonal moments are described in Section 2. Experimental results are showed and discussed in Section 3. Finally the conclusions of the research are reported in Section 4.

\section{Orthogonal Moments}

Moments can be defined as scalar quantities used to characterize a function and to capture its significant features. We can define a moment in a general way as

$$
M_{p q}=\iint_{D} P_{p q}(x, y) f(x, y) d x d y
$$

where $p, q$ are non-negative integers and $p+q$ is called the moment order. $f(x, y)$ is the image intensity function, and $\left\{P_{00}(x, y), P_{01}(x, y), \ldots, P_{i j}(x, y)\right\}$ are polynomial basis functions defined on $D$.

Depending on the used polynomial basis function various systems of moments can be formed. By far, the most popular type of moments is the geometric, which uses as polynomial basis the standard power basis $\left\{x^{i} y^{j}\right\}$. Based on geometric moments, $\mathrm{Hu}$ [6] introduced a group of seven moment invariants that have also been used as features because of their rotation, scaling and translation invariance.

However, the basis set employed in geometric moments is not orthogonal. Therefore, they are not optimal with respect to the information redundancy and it is very difficult to recover an image from them [1]. In order to overcome this problem, Teague [7] suggested the use of orthogonal moments that are defined in terms of continuous orthogonal polynomials. This is the case of Legendre [1] and Zernike [8]. Another advantage of orthogonal moments is that they commonly have a low computational complexity because, we can evaluate them by using recurrent relations [9]. Hence, if the polynomial basis is orthogonal and satisfies the following condition of orthogonality: 


$$
\iint_{\Omega} P_{p q}(x, y) \cdot P_{m n}(x, y) d x d y=0
$$

for any indexes $p \neq m$ and $q \neq n$, we can say that we are in the presence of orthogonal moments where $\Omega$ is the orthogonality area.

Two important cases of orthogonal polynomial bases that can be used for constructing moments are the following. The first one consists in taking the direct product of two systems of orthogonal polynomials in one variable. Let $\left\{P_{p}(x)\right\}$ and $\left\{Q_{q}(y)\right\}$ be the orthogonal polynomial bases defined on the sets $\Omega_{1}$ and $\Omega_{2}$ respectively, with $\Omega=\Omega_{1} \times \Omega_{2}$. Then,

$$
P_{p q}(x, y)=P_{p}(x) Q_{q}(y)
$$

is the orthogonal polynomial of degree $p+q$ defined on $\Omega$. The second case, also called radial orthogonal functions, uses a unit disc, $\left\{(x, y): x^{2}+y^{2} \leq 1\right\}$, as orthogonality area. Its general form is the following:

$$
P_{p q}(x, y)=R_{p q}(\rho) e^{j q \theta}
$$

where $\rho=x^{2}+y^{2}, \theta=\arctan (y / x)$, and $R_{p q}(\rho)$ is a polynomial in $\rho$. One advantage of this form is that it is invariant with respect to rotation of axes. An example of radial orthogonal functions used for moments construction are Zernike [8], pseudo-Zernike [9] and Generalized pseudo-Zernike [10].

Besides, continuous and discrete functions can be used as polynomial bases. The use of continuous functions like Legendre [1], Gegenbauer [9], and the Zernike family, requires the transformation of the image coordinates space and an approximation of the integrals, causing some numerical errors. This problem can be avoided by using discrete orthogonal polynomials as basis functions, which eliminates the need for numerical approximation. They also exactly satisfy the orthogonal property in the discrete domain of image coordinate space [5]. Example of discrete moments are Tchebichef [11], Krawtchouk [3], Hahn [12], dual Hahn [4] and Racah [5].

\section{Experimental Results}

In this study, we made three kinds of experiments. Each one involves the training of a Support Vector Machine (SVM) [13] with a group of digit images. The SVM was configured to employ a lineal kernel and the parameters were optimally selected by using cross-validation. A second group of digit images were used to predict their labels using SVM, once it was trained.

Two data sets of digit images were used. The first one is composed by 3700 printed digit images taken from Cuban license plates images. And the second one is composed by 3200 handwritten digit images scanned from envelopes by the U.S. Postal Service [14]. Each data set was divided in two groups, one for training and the other for testing. In both data sets the image size is $30 \times 30$ pixels. In the case of handwritten data set, different handwritten styles were taken into consideration influencing the character figure form, size, thickness and orientation. While the printed data set was 


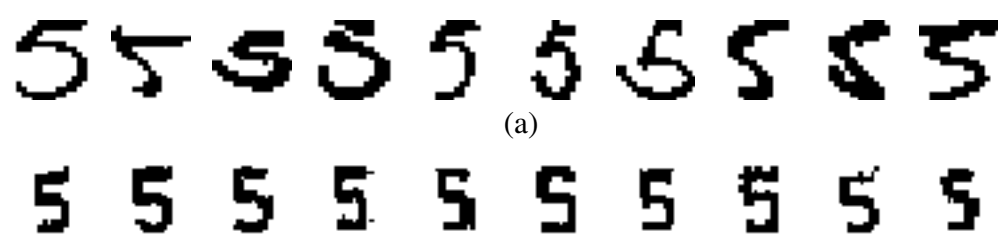

(b)

Fig. 1. Sample set of handwritten (a) and printed (b) digits images used in the experiments

constituted by images with different complexity degree, caused by segmentation and binarization errors. In addition, the used font makes different classes like $\{5,6,8,9\}$ share some common strokes, making more difficult their classification.

The criteria used for measuring the results are the classification precision $(\mathrm{P})$, recall (R) and accuracy (A).

$$
P=\frac{t p}{t p+f p} \quad R=\frac{t p}{t p+f n} \quad A=\frac{t p+t n}{t p+t n+f p+f n}
$$

were $t p$ stands for true positive, $f p$ false positive, tn true negative and $f n$ false negative.

In the next three sections, we present the experiment that we made in this study.

\subsection{Comparison of Feature Extraction Methods}

In this experiment we compare a group of nine orthogonal moments against other feature extraction methods such as geometric moments, Hu invariants, curvature approximation (CA), standard (SFD) and elliptic (EFD) Fourier descriptors.

As it can be seen from Figs. 2 and 3, that orthogonal moment perform better for classification tasks that other feature extraction method like Fourier descriptors, curvature approximation and geometric moments. Most of them perceive precision results over the 0.90 in the case of handwritten digit recognition, and 0.95 for printed digit recognition. Notice that in this case, all discrete orthogonal moments show very good results, except Hahn moments. In the case of continuous moments, Zernike and pseudo Zernike do not bring good results.

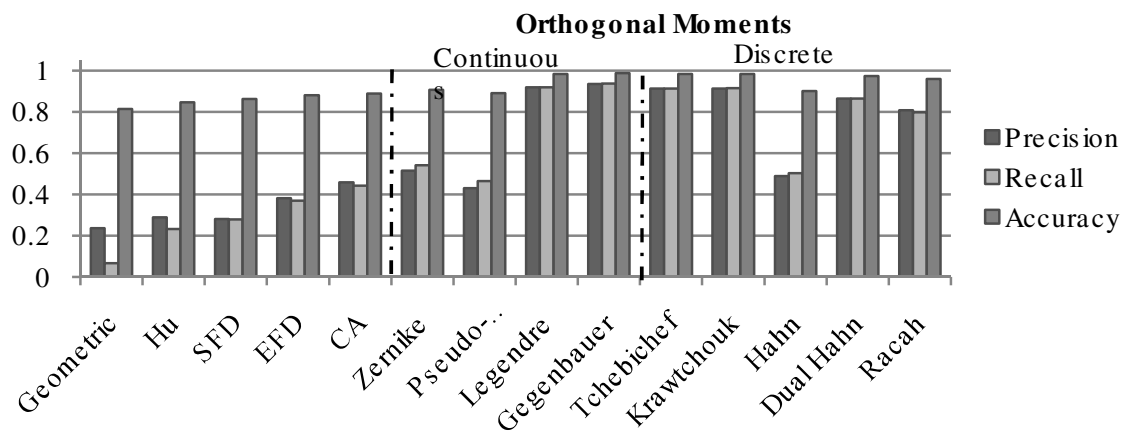

Fig. 2. Comparative analysis of feature extraction methods for handwritten digits classification 


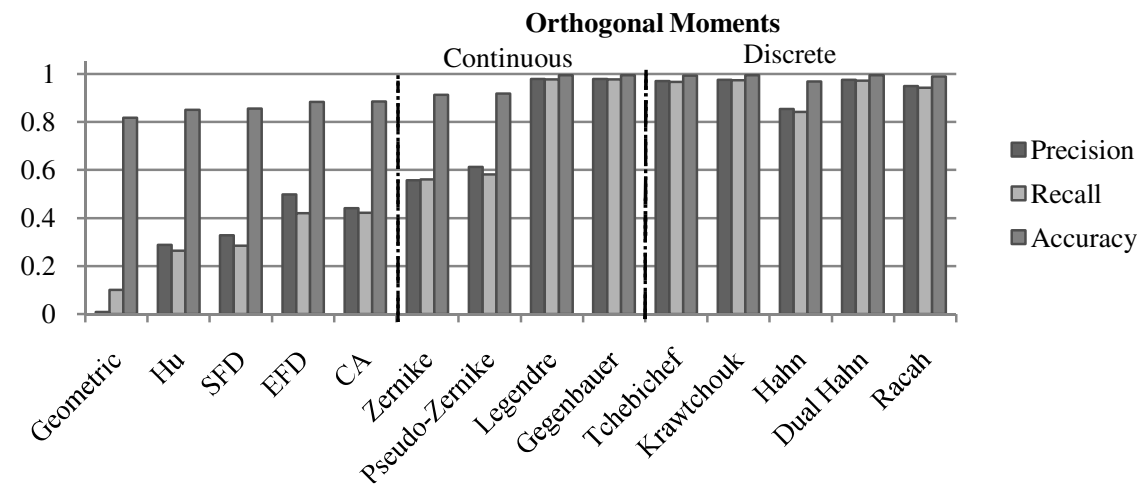

Fig. 3. Comparative analysis of feature extraction methods for printed digits classification

\subsection{Discriminating Capability of Orthogonal Moments}

This experiment measures the discriminating ability of orthogonal moments. They were used as feature extraction methods to solve 5 classification problems, each one with different number of classes. Digits that can be easily misclassified were grouped in the problems with fewer classes. Finally, the class groupings are: $\{2,7\},\{5,6,8,9\}$, $\{2,5,6,7,8,9\},\{0,3,2,5,6,7,8,9\},\{0,1,2,3,4,5,6,7,8,9\}$. The maximum moment order used was 10 .

In Table 1 and Table 2 the results of the experiments are summarized. It can be seen that in the case of handwritten digits classification, Gegenbauer moments perform better in problems with more than 5 classes, while Racah, dual Hahn and Tchebichef moments, do it in the problems with no more than 5 classes.

On the other hand, in the case of printed digits classification, dual Hahn moments obtained the best result for all the problems. However, it is worth mentioning the

Table 1. Comparison of the discriminating ability for handwritten character recognition

\begin{tabular}{|c|c|c|c|c|c|c|c|c|c|c|c|c|c|c|c|c|c|}
\hline & \multirow{2}{*}{$\begin{array}{l}\text { Orthogonal } \\
\text { Moment }\end{array}$} & \multicolumn{3}{|c|}{2 Classes } & \multicolumn{3}{|c|}{4 Classes } & \multicolumn{4}{|c|}{6 Classes } & \multicolumn{3}{|c|}{8 Classes } & \multicolumn{3}{|c|}{10 Classes } \\
\hline & & $\mathrm{P}$ & $\mathrm{R}$ & A & $P$ & $\mathrm{R}$ & A & $\mathrm{P}$ & $\mathrm{R}$ & A & 4 & $\mathrm{P}$ & $\mathrm{R}$ & A & $\mathrm{P}$ & $\mathrm{R}$ & $\mathrm{A}$ \\
\hline \multirow{4}{*}{ 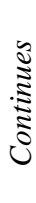 } & Zernike & 0.72 & 0.12 & .12 & 0.65 & 0.0 & 0.80 & 0.53 & 0.5 & 30.8 & 840 & .54 & 0.54 & 0.89 & 0.51 & 0.5 & 0.91 \\
\hline & P. Zernike & 0.73 & .73 & 0.12 & 0.59 & 0.5 & 0.80 & 0.44 & 0.4 & 50.8 & 810 & .46 & 0.45 & 0.86 & 0.43 & 0.46 & 0.89 \\
\hline & Legendre & 0.98 & .98 & 9.9 & 0.94 & 0.9 & 0.97 & 0.93 & 0.9 & 30.9 & 980 & .92( & 0. & 0.3 & 0.92 & 007 & 0.98 \\
\hline & Gegenbauer & 0.98 & 0.98 & 0.9 & 0.97 & 0.9 & 0.9 & 0.95 & 0.9 & 50.9 & 980 & .940 & 0.94 & 0.98 & 0.93 & 0.94 & 0.99 \\
\hline \multirow{5}{*}{ 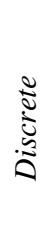 } & Tchebichef & 0.98 & & & .9 & & 0.3 & 0.93 & 0.93 & .9 & 98 & .92( & 0.92 & 0.98 & 0.91 & 0.9 & 0.98 \\
\hline & awtchouk & 0.98 & 0.9 & 0.9 & 0.94 & 0.9 & 0.97 & 0.93 & 0.9 & 30.9 & 980 & 0.910 & 0.91 & 0.98 & 0.91 & 0.9 & 0.98 \\
\hline & Hahn & 0.94 & 093 & 094 & 060 & 04 & 07 & 056 & 05 & 8 & 0 & ( & 0.4 & 0.8 & 0 & 0 & 0.90 \\
\hline & Dual Hahn & 0.98 & 0.98 & 0.98 & 0.95 & 00 & 0.98 & 0.90 & 0.90 & 00.9 & 970 & .87( & 0.87 & 0.97 & 0.86 & 0.86 & 0.97 \\
\hline & Racah & 0.99 & 0.99 & 0.99 & 0.94 & 0.9 & 0.9 & 0.87 & 0.8 & 70.9 & 960 & .72( & 0.73 & 0.93 & 0.81 & 0.80 & 0.96 \\
\hline
\end{tabular}


Table 2. Comparison of the discriminating ability for printed character recognition

\begin{tabular}{|c|c|c|c|c|c|c|c|c|c|c|c|c|c|c|c|c|}
\hline \multirow{2}{*}{\multicolumn{2}{|c|}{$\begin{array}{c}\text { Orthogonal } \\
\text { Moment }\end{array}$}} & \multicolumn{3}{|c|}{2 Classes } & \multicolumn{3}{|c|}{4 Classes } & \multicolumn{3}{|c|}{6 Classes } & \multicolumn{3}{|c|}{8 Classes } & \multicolumn{3}{|c|}{10 Classes } \\
\hline & & $\mathrm{P}$ & $\mathrm{R}$ & A & $\mathrm{P}$ & $\mathrm{R}$ & A & $\mathrm{P}$ & $\mathrm{R}$ & A & $\mathrm{P}$ & $\mathrm{R}$ & A & $\mathrm{P}$ & $\mathrm{R}$ & A \\
\hline \multirow{4}{*}{ 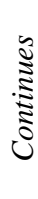 } & Zernike & 0.92 & 0.91 & 0.92 & 0.66 & 0.63 & 0.81 & 0.58 & 0.56 & 0.85 & 0.62 & 0.58 & 0.89 & 90.56 & 0.56 & 0.91 \\
\hline & P. Zernike & 0.94 & 0.84 & 0.94 & 0.60 & 0.60 & 0.79 & 0.61 & 0.61 & 0.87 & 0.63 & 0.57 & 0.89 & 0.61 & 0.58 & 0.92 \\
\hline & Legendre & 0.98 & 0.98 & 0.98 & 0.97 & 0.97 & 0.98 & 0.97 & 0.97 & 0.99 & 0.97 & 0.97 & 0.99 & 0.98 & 0.98 & 1.00 \\
\hline & Gegenbauer & 0.98 & 0.98 & 0.98 & 0.97 & 0.98 & 0.99 & 0.97 & 0.97 & 0.99 & 0.97 & 0.97 & 0.99 & 0.98 & 0.98 & 1.00 \\
\hline \multirow{5}{*}{ 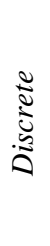 } & Tchebichef & 0.96 & 0.9 & 0.96 & 0.96 & 0.9 & 0.98 & 0.96 & 0.9 & 0.99 & 0.97 & 0.9 & 0.99 & 0.97 & 0.9 & 0.99 \\
\hline & chouk & 0.99 & 0. & 0.99 & 0.99 & 0.99 & 0.99 & 0.98 & 0.98 & 0.99 & 0.97 & 0.97 & 0.99 & 0.98 & 0.9 & 0.99 \\
\hline & Hahn & 0.97 & 0.9 & 0.96 & 0.91 & 0.5 & 0.95 & 0.92 & 0.91 & 0.97 & 0.80 & 0.78 & 0.94 & 40.86 & 0.84 & 0.97 \\
\hline & Dual Hahn & 0.99 & 0.9 & 0.99 & 0.99 & 0.9 & 0.99 & 0.99 & 0.99 & 1.00 & 0.98 & 0.98 & 0.99 & 0.98 & 0.97 & 0.99 \\
\hline & Racah & 0.96 & 0.9 & 0.96 & 0.97 & 0.9 & 0.98 & 0.96 & 0.96 & 0.99 & 0.96 & 0.9 & 0.99 & 0.95 & 0.94 & 0.99 \\
\hline
\end{tabular}

good results of the Krawtchouk moment in the classification problems with a small number of classes, as well as the Legendre and Gegenbauer moments for the ten classes problem.

\subsection{Effect of Moment Order in Classification Results}

The goal of this experiment is to determine the effect of the moments order in the classification results. Handwritten and printed digit images were classified using different orders for the orthogonal moments involved in the experiment. Fig. 4 and 5 shows the results.

From the graphic we can see that high moments order can affect the precision in a drastic way for Tchebichef, dual Hahn and Racah moments in both cases, handwritten and printed digit recognition. It can be caused because of the numerical instability produced by the high moment order. Other moments like Legendre and Zernike can

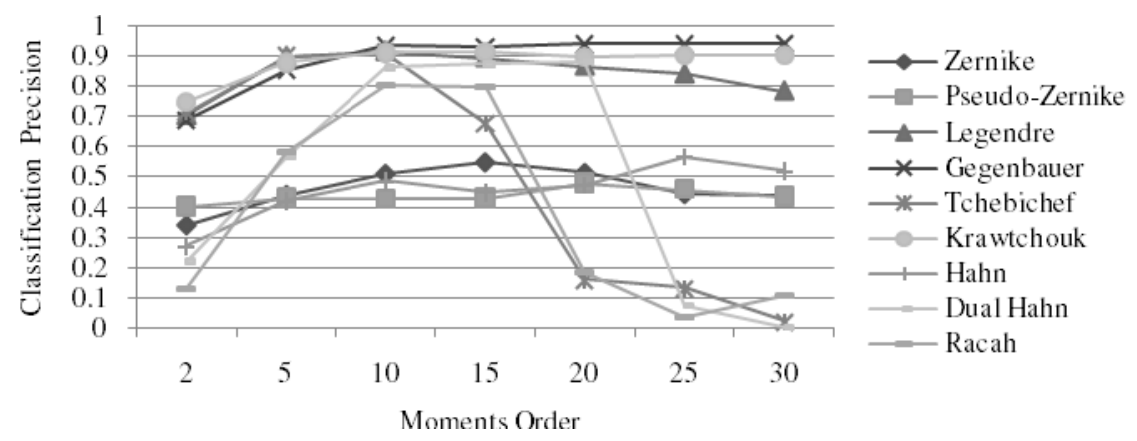

Fig. 4. Classification of handwritten digits using different moments orders 


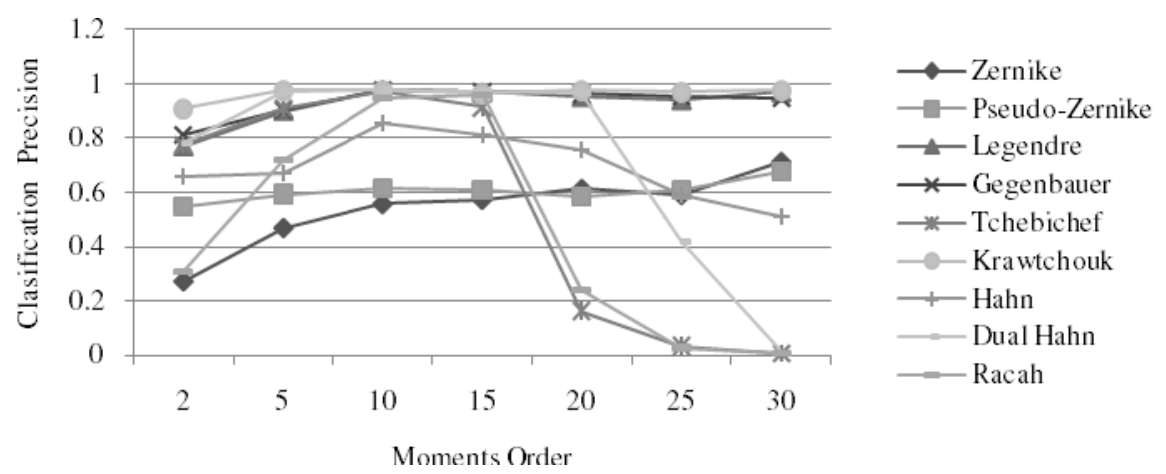

Fig. 5. Classification of printed digits using different moments orders

be affected in a minor way by the increase of the order for the handwritten recognition and the Hahn for the printed digit recognition.

The remaining moments increase the precision as the moment order is increased.

\section{Conclusion}

In this paper, we carried out an experimental comparison of the accuracy of several orthogonal moments as feature extraction methods in character recognition problems. Experimental results showed that orthogonal moments perform better than other more conventional feature extraction techniques such as: geometric moments, Hu invariants and Fourier descriptors, for character images classification The continuous moments Legendre and Gegenbauer have proven to describe very well image features for a classification task, especially in problems with more than 5 classes. However, discrete orthogonal moments like Krawtchouk and Dual Hahn describe better the images in classification problems with no more than 5 classes.

On the other hand, numerical instability, caused by high moment orders, affects drastically the image feature representation of some discrete orthogonal moments like Tchebichef, Dual Hahn and Racah while others like Krawtchouk and Gegenbauer perform better as the moment order increases.

This study corroborates the fact that orthogonal moments as feature extraction methods are, in general, a powerful tool for character recognition problems. However, the optimal orthogonal moment could vary from one problem to another due to the particular characteristics of the problems and the intrinsic properties of each moment. This way, the comparative results presented in this paper can be useful for the selection of a suitable feature extraction method according to the characteristics of the problem at hand.

\section{References}

1. Cheriet, M., Kharma, N.: Character recognition systems: A guide for students and practitiones. Wiley Interscience, Hoboken (2007)

2. Downton, A., Impedovo, S.: Progress in Handwriting Recognition. World Scientific, Singapore (1997) 
3. Yap, P.: Image Analysis by Krawtchouk Moments. IEEE Transaction on Image Processing 12(11), 1367-1377 (2003)

4. Zhu, H., Shu, H.: Image analysis by discrete orthogonal dual Hahn moments. Pattern Recognition Letters 28, 1688-1704 (2007)

5. Zhu, H., Shu, H.: Image analysis by discrete orthogonal Racah moments. Signal Processing 87, 687-708 (2007)

6. Hu, M.: Visual pattern recognition by moment invariants. IRE Trans. Info. Theory, IT 8 , 179-187 (1962)

7. Teague, M.R.: Image analysis via the general theory of moments. J. Opt. Soc. Am. 70, 920-930 (1980)

8. Khotanzad, A., Hong, Y.: Invariant image recognition by Zernike moments. IEEE Transactions on Pattern Analysis and Machine Intelligence 9(1), 489-490 (1990)

9. Flusser, J.: Moments and moments invariants for pattern recognition. Wiley Interscience, Hoboken (2009)

10. Xia, T., Zhu, H.: Image description with generalized pseudo-Zernike moments. Journal of the Optical Society of America 24(1), 50-59 (2007)

11. Mukundan, R.: Image analysis by Tchebichef moments. IEEE Image Analysis by Tchebichef Moments 10(9), 1357-1364 (2001)

12. Zhou, J., Shu, H.: Image analysis by discrete orthogonal Hahn moments. In: Kamel, M.S., Campilho, A.C. (eds.) ICIAR 2005. LNCS, vol. 3656, pp. 524-531. Springer, Heidelberg (2005)

13. Vapnik, V.: The Nature of Statistical Learning Theory. Springer, New York (1995)

14. LeCun, Y., et al.: Handwritten Digit Recognition: Applications of Neural Net Chips and Automatic Learning. IEEE Communication, 41-46 (1989) 